\title{
Dynamics of Biologically Active Subpopulations of Influenza Virus: Plaque-Forming, Noninfectious Cell-Killing, and Defective Interfering Particles ${ }^{\nabla}$
}

\author{
Philip I. Marcus, * John M. Ngunjiri, and Margaret J. Sekellick \\ Department of Molecular and Cell Biology, and Center of Excellence for Vaccine Research, University of Connecticut, \\ Storrs, Connecticut 06269
}

Received 31 December 2008/Accepted 22 May 2009

\begin{abstract}
The dynamic changes in the temporal appearance and quantity of a new class of influenza virus, noninfectious cell-killing particles (niCKP), were compared to defective interfering particles (DIP). After a single high-multiplicity passage in MDCK cells of an egg-derived stock that lacked detectable niCKP or DIP, both classes of particles appeared in large numbers $\left(>5 \times 10^{8} / \mathrm{ml}\right)$, and the plaque-forming particle (PFP) titer dropped $\sim 60$-fold. After two additional serial high-multiplicity passages the DIP remained relatively constant, the DIP/niCKP ratio reached 10:1, and the PFP had declined by about 10,000-fold. Together, the niCKP and DIP subpopulations constituted ca. $20 \%$ of the total hemagglutinating particle population in which these noninfectious biologically active particles (niBAP) were subsumed. DIP neither killed cells nor interfered with the cell-killing (apoptosis-inducing) activity of niCKP or PFP (infectious CKP), even though they blocked the replication of PFP. Relative to the UV-target of $\sim 13,600$ nucleotides (nt) for inactivation of PFP, the UV target for niCKP was $\sim 2,400 \mathrm{nt}$, consistent with one of the polymerase subunit genes, and that for DIP was $\sim 350 \mathrm{nt}$, consistent with the small DI-RNA responsible for DIP-mediated interference. Thus, niCKP and DIP are viewed as distinct particles with a propensity to form during infection at high multiplicities. These conditions are postulated to cause aberrations in the temporally regulated replication of virus and its packaging, leading to the production of niBAP. DIP have been implicated in the virulence of influenza virus, but the role of niCKP is yet unknown.
\end{abstract}

Infectious particles constitute a minor subpopulation of biologically active influenza virus populations but command major attention because of their critical role in replication and pathogenesis. However, there are other subpopulations of biologically active particles (BAP). Some of these particles, such as interferon (IFN)-inducing particles (IFP) (20), IFN induction-suppressing particles (ISP) (21), or defective interfering particles (DIP) (26), are intrinsically noninfectious and exist in large excess over infectious virions. These noninfectious BAP (niBAP) have the potential to influence the course of pathogenesis through their capacity to stimulate or suppress antiviral responses intrinsic to the innate immune system $(8,11,14,30$, $34,42)$ and/or by direct interference with virus replication (26). Noninfectious cell-killing particles (niCKP) of influenza virus represent a newly identified member of the niBAP family (29). The numbers and sizes of these subpopulations and their contribution to pathogenesis are poorly understood because the extent to which they appear and function in a population of host cells, let alone during natural infection $(4,9)$, has not been assessed. All subpopulations of infectious and noninfectious BAP are subsumed in the population of hemagglutinating particles (HAP) which represent total physical particles. Although the majority of HAP have no known biological activity, they are deemed capable of contributing large numbers of gene

\footnotetext{
* Corresponding author. Mailing address: Department of Molecular and Cell Biology, University of Connecticut, U-3125, 91 North Eagleville Rd., Storrs, CT 06269. Phone: (860) 486-4254. Fax: (860) 486-5193. E-mail: philip.marcus@uconn.edu.

${ }^{\nabla}$ Published ahead of print on 3 June 2009.
}

segments to cells in the course of infection. It is not known whether such a large influx of gene copies can compromise the normal temporal regulation of virus replication $(24,38)$ and the proper packaging of gene segments $(16,33)$, thereby influencing the generation of niBAP and the outcome of an infection or the action of live-attenuated vaccines $(14,34,39,42)$.

Considerable progress has been reported in identifying genetic changes within infectious particles of influenza virus that directly affect its virulence $(3,43)$. What is less clear is the extent to which the large subpopulations of niBAP and biologically inactive HAP that may be presented to cells during the course of infection contribute to virulence and pathogenesis.

This report compares for the first time the generation of subpopulations of niCKP (29) under conditions of high-multiplicity (HM) passages that also favor the generation of DIP, the classical von Magnus particles (41) that interfere with virus replication (26), and act as an antiviral agent (12). Production of DIP is most often associated with HM passages of the virus $(26,41)$. Evidence is provided here that niCKP and DIP represent two distinct subpopulations of influenza virus and that niCKP share the attributes of defective noninterfering particles (DNIP) inferred from an observed excess of polymerase activity relative to that expected on the basis of infectivity in influenza virus stocks (7). Lastly, a model is proposed showing a transitional state for subpopulations of BAP from the most to the least complex and that postulates their origin, in part, from aberrations that may result from high multiplicities during infection. 


\section{MATERIALS AND METHODS}

Cell culture and media. GMK-Vero and MDCK cells were grown in attachment solution (AS; NCI medium plus $6 \%$ calf serum) $(29,37)$ and minimal essential medium plus $5 \%$ calf serum, respectively, and incubated at $37.5^{\circ} \mathrm{C}$ with a flowing air- $\mathrm{CO}_{2}$ mixture to maintain $\mathrm{pH}$ 7.1. Primary chicken embryo kidney cells (CEK) were prepared from 18-day-old embryos and obtained from Charles River Specific-Pathogen-Free Avian Supplies (SPAFAS, Inc., Storrs, CT) and also grown in AS.

Viruses: source and preparation. Seed stocks of low-pathogenicity influenza viruses were provided by colleagues as follows: A/TK/WI/68 (H5N9), A/CK/PA/ 13609/93 (H5N2), A/TK/OR/71-SEPRL (H7N3)-NS1 $1_{1-230}$, and A/TK/OR/71delNS1 $1_{1-124}(\mathrm{H} 7 \mathrm{~N} 3)$ were obtained from D. L. Suarez, Southeast Poultry Research Laboratory, Athens, GA; rgA/PR/8/34 (H1N1) was obtained from R. G. Webster, St. Jude Children's Research Hospital, Memphis, TN; A/PR/8/ 34(H1N1) was obtained from A. Garcia-Sastre and P. Palese, Mt. Sinai School of Medicine, New York, NY; A/CK/CT/72/2003 (H7N2) was obtained from the USDA, National Veterinary Services Laboratories, Ames, IA; and B/MD/59 was obtained from R. M. Krug, University of Texas, Austin, TX.

A/TK/OR/71-SEPRL encodes a full-length NS1 (amino acids 1 to 230), and $\mathrm{A} / \mathrm{TK} / \mathrm{OR} / 71$-delNS1 $1_{1-124}$ expresses a truncated NS1 protein (amino acids 1 to 124) $(8,31)$. These two variants are here designated $\mathrm{NS}_{1-230}$ and delNS1 $1_{1-124}$ respectively. A/TK/OR/71 clones 1 and 2 were isolated from plaques of variant $\mathrm{NS}_{1-230}$ in CEK and MDCK cells, respectively. Stocks of influenza viruses were propagated in 9-day-old specific-pathogen-free embryonated chicken eggs (SPAFAS). Each egg was injected with $0.1 \mathrm{ml}$ containing $\sim 10^{3} \mathrm{PFP}$, incubated for 48 to $72 \mathrm{~h}$ at $34^{\circ} \mathrm{C}$ with forced humidified air circulation and egg rotation, and cooled for $24 \mathrm{~h}$ at $4^{\circ} \mathrm{C}$ before harvesting. The chorioallantoic fluid was harvested and stored separately for each egg at $-80^{\circ} \mathrm{C}$. Fluids with high hemagglutinating activity (hemagglutinin $[\mathrm{HA}]$ titers $\geq 1,280$ ) were pooled, divided into aliquots, and stored at $-80^{\circ} \mathrm{C}(21)$. Vesicular stomatitis virus (VSV) and Sindbis virus were grown in confluent monolayers of Vero cells (36).

PFP assay. Plaque-forming particles (PFP) were assayed as described previously (29), either in CEK without trypsin in the overlay or in Vero cells with a serum-free $0.6 \%$ agarose overlay containing trypsin (T14263 [Sigma]; $1.5 \mu \mathrm{g} / \mathrm{ml}$ ). A second layer of trypsin-agarose was added $24 \mathrm{~h}$ later (44). Development of countable plaques required 1 to 2 days and 3 to 5 days of incubation at $37.5^{\circ} \mathrm{C}$ for CEK and Vero cells, respectively. Both cell systems gave similar plaque titers. CEK cells were used in most of the assays. Sindbis virus and VSV were assayed in Vero cells as described above except that the overlay did not contain trypsin.

CKP assay. A clonogenic (single-cell plating) assay was used to determine the number of influenza virus particles capable of killing cells (29). Briefly, confluent monolayers of GMK-Vero cells $\left(2 \times 10^{6}\right.$ cells/50-mm dish $)$ were challenged with various doses of influenza virus. After virus attachment the monolayers were washed with NCI medium and monodispersed with EDTA-trypsin. Suspensions of single cells were seeded into $50-\mathrm{mm}$ dishes in $5 \mathrm{ml}$ of AS to allow the formation of a countable number of visible colonies after 9 to 10 days of undisturbed incubation. Colonies were fixed, stained, and counted to determine the fraction of cells that survived virus infection relative to that of uninfected control cells (29). CKP multiplicity $\left(\mathrm{m}_{\mathrm{ckp}}\right)$ was calculated from cell survival curves generated as a function of virus concentration. The dilution of virus that produced $0.37(37 \%)$ surviving cells was assumed to contain $\mathrm{m}_{\mathrm{CKP}}=1$ based on a Poisson distribution of CKP in the cell monolayer (29). PFP are infectious particles that kill cells and thus are also termed infectious CKP (iCKP). The difference between the total CKP population and the PFP(iCKP) subpopulation represents the niCKP subpopulation.

HAP assay. In order to relate the number of physical particles of influenza virus to $\mathrm{BAP}$, the HA titers (hemagglutination units $[\mathrm{HAU}] / \mathrm{ml}$ ) were expressed as $\mathrm{HAP} / \mathrm{ml}$ by using a factor that converted HAU into physical particles scored as HAP. Several groups have determined this conversion factor independently by measuring the number of physical particles of influenza virus in $1 \mathrm{HAU}$. We have averaged the conversion factors from six different reports, as cited in more recent studies $(10,13,25)$. When the final concentration of chicken red blood cells in each tube of the HA assay was $10^{7} / \mathrm{ml}$, the highest dilution of virus that gave complete hemagglutination was considered to contain 1 HAU. The following relationship was used: [(HA titer in $\mathrm{HAU} / \mathrm{ml}) \times\left(5 \times 10^{6}\right.$, the conversion factor $)=$ the number of $\mathrm{HAP} / \mathrm{ml}$.

Generation of DIP stocks. Stocks of high-titer DIP were generated or amplified by HM passage of influenza virus in CEK or MDCK cells. The seed viruses usually were stocks propagated by low-multiplicity (LM) passage in chicken embryonated eggs. The virus material, usually $\geq 1,000 \mathrm{HAU} / \mathrm{ml}$, i.e., the equivalent of $\geq 5 \times 10^{9} \mathrm{HAP} / \mathrm{ml}$, was diluted 1:2 in AS and attached to cell monolayers for $1 \mathrm{~h}$ at $37.5^{\circ} \mathrm{C}$ and washed twice with $2 \mathrm{ml}$ of medium without serum, and $2 \mathrm{ml}$ of the medium was added back to the cells. The medium was harvested after 24 to $48 \mathrm{~h}$ of incubation at $37.5^{\circ} \mathrm{C}$ and cleared of cell debris by centrifugation at $2,000 \times g$ for $10 \mathrm{~min}$. The ratio of HAP to PFP in these samples typically increased with the number of passages and peaked by the third passage. However, the HAP/PFP ratio in several isolates that contained measurable DIP after LM passage (see Results) peaked in the first passage. HM passages of virus were regularly used as the standard DIP stocks unless stated otherwise.

DIP assay. DIP activity was measured by PFP yield reduction of a standard (helper) virus. A/TK/OR/71-NS1(1-124) was $\sim 3$ times more sensitive to DIP action than any of the three other viruses tested and hence was used as the standard virus for all DIP assays. Importantly, this virus does not contain measurable niCKP or DIP, thereby providing a low basal level of particles that might affect PFP yield reduction assays. Monolayers containing $2.4 \times 10^{6}$ MDCK cells in 50-mm dishes were simultaneously infected with standard virus at an $\mathrm{m}_{\mathrm{PFP}}$ of 0.3 and increasing amounts of the sample being tested for DIP. Virus was attached for $1 \mathrm{~h}$, followed by two washes with serum-free medium. A portion ( 2 $\mathrm{ml}$ ) of fresh serum-free medium that contained $1.5 \mu \mathrm{g}$ of trypsin/ml was added, and the plates were incubated for $24 \mathrm{~h}$ at $37.5^{\circ} \mathrm{C}$. The medium then was harvested, centrifuged to remove cell debris, and assayed for PFP. The data were plotted as the fraction of surviving PFP from standard virus as a function of increasing amounts of the samples being tested for DIP content. This assay is similar to that used by Bellett and Cooper to measure VSV DIP (5) and, in principle, should give the same results as the infectious-center reduction assay used for influenza virus (18). According to a Poisson distribution of DIP in the monolayer, the dilution of virus that resulted in $37 \%$ survival of PFP contained a multiplicity of 1 DIP per cell. DIP titers were then calculated as a product of the $\mathrm{m}_{\mathrm{DIP}}$, dilution factor, and the number of cells in the monolayer (22).

DIP action on CKP. Several LM-passage viruses were found to contain PFP(iCKP), niCKP and DIP, raising the question as to whether DIP can block $\mathrm{CKP}$ activity while preventing virus replication. Monolayers of Vero cells were coinfected with the standard virus $\left[\mathrm{m}_{\mathrm{PFP}(\mathrm{iCKP})}=3\right]$ and DIP $\left(\mathrm{m}_{\mathrm{DIP}}=5\right)$. After virus attachment and washing, a soft agarose overlay $(0.3 \%)$ with no trypsin was added immediately to localize apoptotic cells when they appeared. The development of apoptosis in the monolayer, with or without DIP present, was observed microscopically and recorded with digital images after incubation at $37.5^{\circ} \mathrm{C}$ for $24 \mathrm{~h}$. Another set of Vero cell monolayers was subjected to a clonogenic assay to determine the fraction of cells killed by standard virus in the presence or absence of DIP.

Sensitivity of PFP, iCKP, niCKP, and DIP to UV radiation. UV target theory posits that a single lethal hit of UV radiation to a gene segment inactivates that segment with respect to a measurable phenotype. Thus, a lethal UV hit to any one of the eight independently transcribed gene segments of influenza virus required for infectivity results in the inactivation of PFP $(1,29)$. DIP was the most resistant to UV radiation among the BAP measured here, as shown originally by Nayak et al. (27) and later confirmed (19). As a consequence, samples being tested for DIP that contained PFP that would interfere with a PFP yield reduction assay were UV irradiated with $390 \mathrm{ergs} / \mathrm{mm}^{2}$ to inactivate $(99.97 \%)$ of the residual PFP and (75\%) of the CKP, with a negligible loss of DIP activity.

\section{RESULTS}

Infectious and noninfectious cell-killing particle subpopulations. The first six isolates of influenza virus listed in Table 1 contained significant amounts of both PFP(iCKP) and niCKP. The PFP(iCKP) titers ranged from $0.45 \times 10^{8}$ to $5.8 \times 10^{8} / \mathrm{ml}$, with a mean of $2.6 \times 10^{8} / \mathrm{ml}$, while those of the niCKP ranged from $3.5 \times 10^{8}$ to $19 \times 10^{8} / \mathrm{ml}$, with a mean of $10 \times 10^{8} / \mathrm{ml}$. Thus, the CKP subpopulations consisted of $\sim 75 \%$ niCKP and $\sim 25 \%$ $\mathrm{PFP}(\mathrm{iCKP})$. Notably, the other three isolates [delNS1 $1_{1-124}, \mathrm{PR} /$ $8 / 34$, and $\mathrm{rgPR} / 8 / 34$ reconstructed from the wild-type virus by using reverse genetics] had little or no detectable niCKP based on the near equivalence of PFP and CKP titers.

Quantification of DIP by PFP yield reduction assay. Four different isolates of influenza virus maintained by LM passages in eggs were screened for use as a standard (helper) virus in a plaque yield reduction assay for DIP detection (5). Confluent monolayers of MDCK cells $\left(2.4 \times 10^{6} / 50-\mathrm{mm}\right.$ plate $)$ were coinfected with the test standard (helper) virus at an $\mathrm{m}_{\mathrm{PFP}}$ of 
TABLE 1. Comparative titers of biologically active particles and HAP in subpopulations of different isolates of influenza virus maintained by LM passages in embryonated eggs

\begin{tabular}{|c|c|c|c|c|c|}
\hline \multirow{2}{*}{ Isolate } & \multicolumn{5}{|c|}{ Titer $(\text { per ml })^{a}$} \\
\hline & $\mathrm{PFP}^{b}$ & Total $\mathrm{CKP}^{c}$ & $\operatorname{niCKP}^{d}$ & DIP $^{e}$ & HAP $^{f}$ \\
\hline TK/OR/71 -NS1(1-230) clone 1 (H7N3) & $5.8 \times 10^{8}$ & $20 \times 10^{8}$ & $14 \times 10^{8}$ & $48 \times 10^{8}$ & $193 \times 10^{8}$ \\
\hline TK/OR/71 -NS1(1-230) clone 2 (H7N3) & $4.7 \times 10^{8}$ & $18 \times 10^{8}$ & $13 \times 10^{8}$ & $48 \times 10^{8}$ & $193 \times 10^{8}$ \\
\hline TK/WI/68 (H5N9) & $0.45 \times 10^{8}$ & $6.5 \times 10^{8}$ & $6.1 \times 10^{8}$ & $24 \times 10^{8}$ & $96 \times 10^{8}$ \\
\hline CK/PA/13609/93 (H5N2) & $0.83 \times 10^{8}$ & $5.6 \times 10^{8}$ & $4.8 \times 10^{8}$ & $22 \times 10^{8}$ & $203 \times 10^{8}$ \\
\hline TK/OR/71 -NS1(1-230) (H7N3) & $3.2 \times 10^{8}$ & $22 \times 10^{8}$ & $19 \times 10^{8}$ & $8.6 \times 10^{8}$ & $307 \times 10^{8}$ \\
\hline $\mathrm{CK} / \mathrm{CT} / 73 / 03(\mathrm{H} 7 \mathrm{~N} 2)$ & $0.7 \times 10^{8}$ & $3.8 \times 10^{8}$ & $3.5 \times 10^{8}$ & $8.6 \times 10^{8}$ & $102 \times 10^{8}$ \\
\hline $\mathrm{PR} / 8 / 34(\mathrm{H} 1 \mathrm{~N} 1)$ & $16 \times 10^{8}$ & $16 \times 10^{8}$ & $<0.1 \times 10^{8 *}$ & $2.0 \times 10^{8}$ & $393 \times 10^{8}$ \\
\hline $\mathrm{rgPR} / 8 / 34(\mathrm{H} 1 \mathrm{~N} 1)$ & $2.7 \times 10^{8}$ & $3.4 \times 10^{8}$ & $0.7 \times 10^{8}$ & $<0.1 \times 10^{8 *}$ & $203 \times 10^{8}$ \\
\hline TK/OR/71 -delNS1(1-124) (H7N3) & $6.9 \times 10^{8}$ & $6.9 \times 10^{8}$ & $<0.1 \times 10^{8 *}$ & $<0.1 \times 10^{8 *}$ & $203 \times 10^{8}$ \\
\hline
\end{tabular}

$a *$, Less than the level of detection by the assay.

${ }^{b}$ Assayed on primary chicken embryo kidney cells.

${ }^{c}$ Measured by clonogenic assay (29).

${ }^{d}$ niCKP $=$ total CKP - PFP.

${ }^{e}$ Assayed as described for Fig. 2.

${ }^{f}$ Calculated from absolute HA assays with chicken red blood cells (see Materials and Methods).

0.3 plus increasing amounts of a DIP stock generated by a HM passage of variant TK/OR/71-NS1(1-230) in CEK cells. The DIP stock was UV irradiated $\left(390 \mathrm{ergs} / \mathrm{mm}^{2}\right)$ to inactivate residual PFP so as not to increase the input $\mathrm{m}_{\mathrm{PFP}}$ of the standard virus. Figure 1 shows that of the four different standard stocks tested, the most responsive to the action of DIP

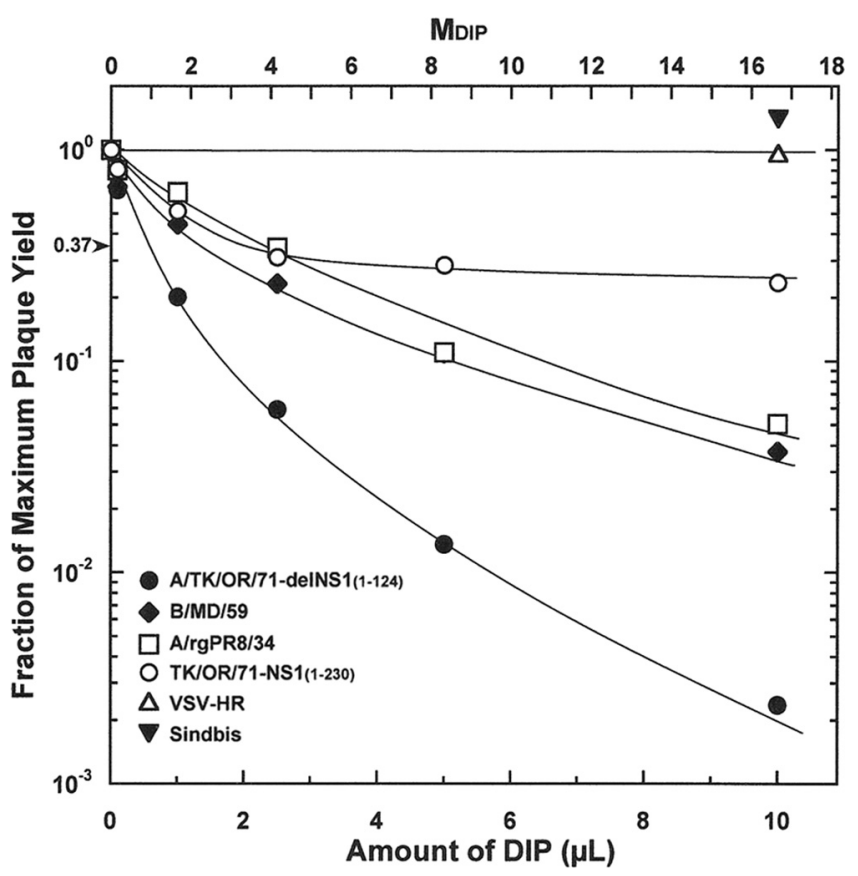

FIG. 1. Effect of DIP on standard influenza virus isolates and heterologous viruses (VSV and Sindbis virus) as measured by PFP yield reduction. A stock of variant TK/OR/71-NS1 $1_{1-230}$ was enriched for DIP by an HM passage in CEK cells. Each monolayer of MDCK was simultaneously infected at an $\mathrm{m}_{\mathrm{PFP}}$ of 0.3 with a test isolate, maintained by LM passages in embryonated chicken eggs (see the symbol key in the figure) and increasing amounts of DIP (lower abscissa). The fraction of maximum PFP yield (ordinate) was plotted as a function of the amount of DIP added (in $\mu \mathrm{l}$ ) per $2.4 \times 10^{6}$ cells. The $\mathrm{m}_{\text {DIP }}$ (upper abscissa) was calculated based on variant delNS1 $1_{1-124}$, which is the most sensitive of the standard viruses. was variant $\mathrm{TK} / \mathrm{OR} / 71-$ delNS1 $1_{1-124}$ (lowest curve). The other three isolates were about one-third as sensitive to DIP action. On this basis, LM passages of variant-delNS1 $1_{1-124}$ were used as standard (helper) virus throughout the present study. The upper abscissa in Fig. 1 shows the values of $\mathrm{m}_{\mathrm{DIP}}$ calculated from the $37 \%$ survival level of the PFP yield reduction curve for variant-delNS1 $1_{1-124}$. Based on a Poisson distribution of DIP among the cell population, the initial exponential loss of PFP activity as a function of DIP concentration indicates a single DIP suffices to block completely the yield of PFP in coinfected cells. This statistically based interpretation (5) was proved to be correct by a direct test of PFP yields from single cells coinfected with DIP and PFP from VSV (36).

During optimization of the DIP assay we corroborated an earlier report that the action of DIP can be partially reversed with increasing multiplicities of standard virus (2; data not shown). Thus, standard virus always was used at an $\mathrm{m}_{\mathrm{PFP}}$ of 0.3 . An added advantage of using LM passages of variant-delNS1 $1_{1-124}$ is the absence of niCKP (Table 1). This eliminates any possible contribution of niCKP to tests for DIP action. As shown in Fig. 1 (uppermost curve), influenza virus-derived DIP did not interfere with the replication of viruses from other families: Rhabdoviridae (VSV) and Togaviridae (Sindbis virus). This lack of heterologous interference is a hallmark of DIP action (26).

Basal levels of DIP in populations of LM passages of influenza viruses. Although the relatively high titer of PFP in LMpassage egg-derived populations of variant NS1(1-230) $(3.2 \times$ $10^{8} / \mathrm{ml}$ ) would appear to preclude DIP in the stock, earlier reports that some influenza virus isolates may naturally contain DIP $(4,9)$ prompted us to test for their presence. Because the high titers of PFP intrinsic to LM passages of virus might mask the detection of DIP (2), we took advantage of the high resistance of DIP to UV radiation as previously reported (27) and first exposed the stocks to $390 \mathrm{ergs}$ of UV radiation $/ \mathrm{mm}^{2}$. This reduced the PFP titer $\sim 10^{4}$-fold with virtually no effect on the titer of DIP (27). HAP titers were unaffected. The residual PFP activity after UV radiation was determined experimentally not to affect the DIP assay (data not shown). Figure 2 shows the results of a DIP assay of TK/OR/71-NS1 $1_{1-230}$ and $\mathrm{NS}_{1-124}$. The lowest abscissa indicates the amount of UV-irradiated 


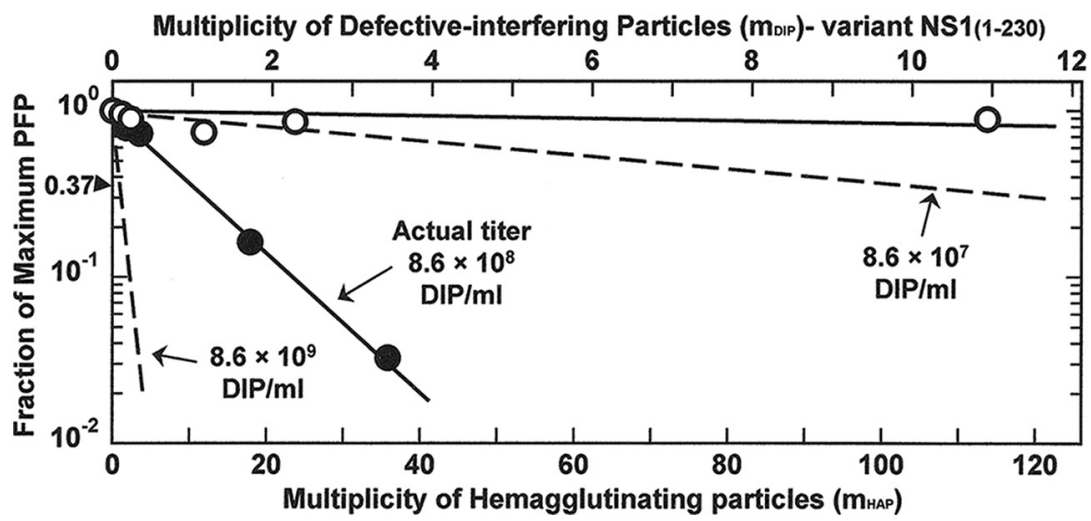

FIG. 2. Assays of DIP in LM-passage stocks of influenza virus TK/OR/71 variants $\mathrm{NS}_{1-230}(\bullet)$ and delNS1 $1_{1-124}(\bigcirc)$. Variant delNS1 $1_{1-124}$ was used as the standard virus. The $\mathrm{m}_{\mathrm{DIP}}$ was calculated for variant $\mathrm{NS1}_{1-230}$ (upper abscissa, closed circles) as described in Materials and Methods. For comparison, theoretical curves are presented for DIP titers that are 10-fold higher (lower dashed line) or 10-fold lower (upper dashed line). The upper curve was generated by variant delNS1(1-124) (open circles) and reveals no detectable DIP. The input virus was standardized to $\mathrm{m}_{\mathrm{HAP}}$ (physical particles).

virus being tested for DIP. The $\mathrm{m}_{\text {DIP }}$ shown in the upper abscissa was calculated from the exponential survival curve for $\mathrm{PFP}$ as a function of $\mathrm{NS}_{1-230}$ multiplicity as described in Materials and Methods. The resulting titer was $8.6 \times 10^{8} \mathrm{DIP} / \mathrm{ml}$. Also presented for comparison are the theoretical PFP yield reduction curves expected (indicated as dashed lines) if the stock of $\mathrm{NS}_{1-230}$ had contained 10 times more $\left(8.6 \times 10^{9} / \mathrm{ml}\right)$ or 10 times less $\left(8.6 \times 10^{7} / \mathrm{ml}\right)$ DIP. A titer of $\sim 10^{7} \mathrm{DIP} / \mathrm{ml}$ approaches the practical lower limit of detection of DIP by this mode of assay. The lower abscissa labeled $\mathrm{m}_{\mathrm{HAP}}$ allows a comparison between the amount of physical particles scored as HAP and DIP. For this stock, the HAP/DIP ratio was $\sim 10$. In marked contrast, NS1(1-124) did not reduce the yield of PFP from standard virus, indicating an absence of detectable DIP (Fig. 2).

The unanticipated detection of DIP in a LM passaged stock of $\mathrm{NS}_{1-230}$ with a high titer of infectious virus prompted us to test for DIP in stocks of other isolates similarly maintained (Table 1). These tests included two plaque-derived (cloned) stocks of $\mathrm{NS}_{1-230}$ in an unsuccessful attempt to obtain populations free of DIP. In total, nine isolates were tested for basal levels of DIP. Table 1 and Fig. 3 show that six of the nine isolates contained DIP in excess of PFP, ranging broadly from $\mathrm{DIP} / \mathrm{PFP}$ ratios of 2.7 to 53 . One of the remaining three isolates had a DIP/PFP ratio of 0.125 , indicating that PFP were eightfold in excess of DIP. The other two isolates had unde-

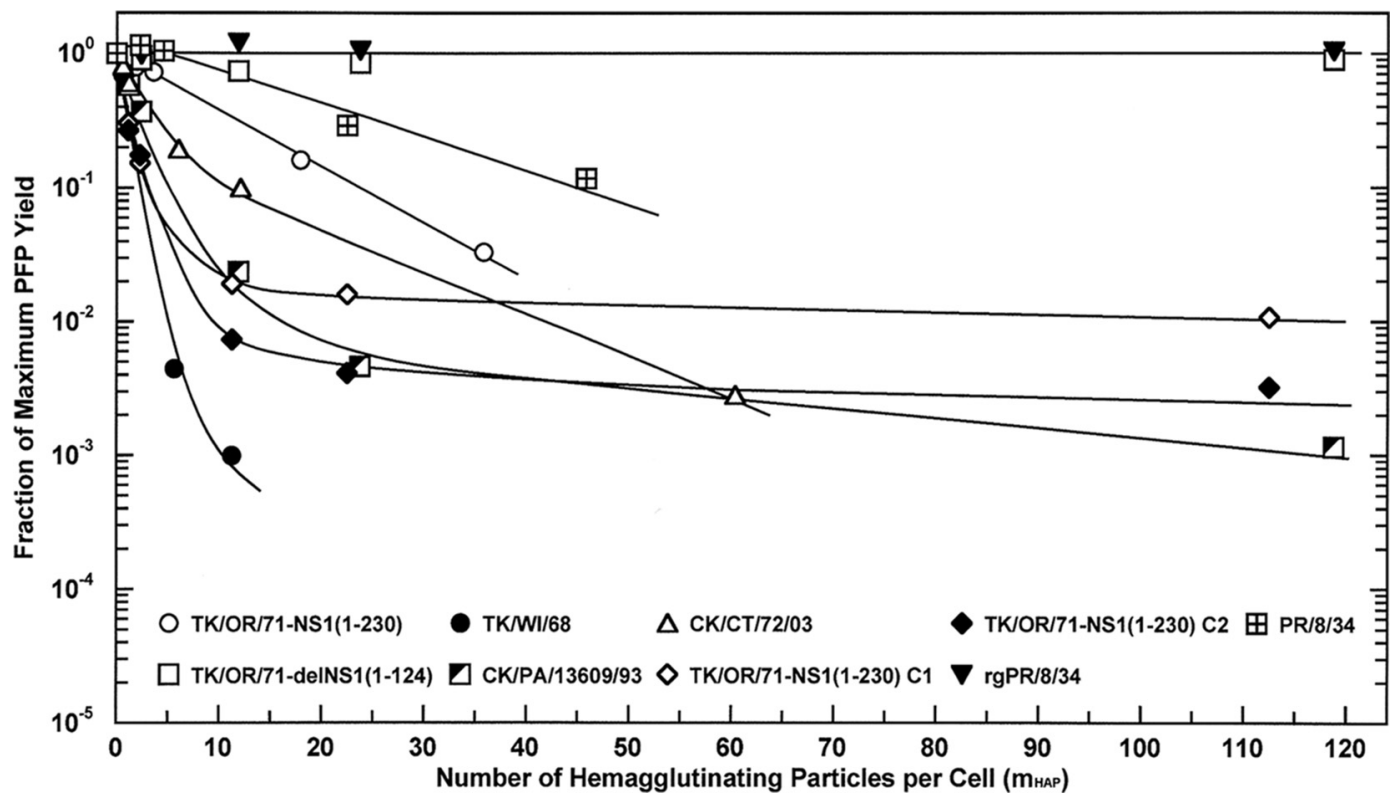

FIG. 3. Detection of DIP in LM-passage stocks of influenza virus isolates. Plaque yield reduction assays for nine different isolates of influenza virus A were carried out as described in the legend of Fig. 1. The amount of each virus added to determine the DIP concentration was standardized in terms of the number of HAP per cell ( $\mathrm{m}_{\mathrm{HAP}}$ ) (abscissa). This allowed a determination of the HAP/DIP ratio. The stocks of the isolates tested contained various quantities of DIP, ranging from undetectable levels (upper two curves; see also Table 1) to some high titers (bottom seven curves; see also Table 1) as indicated by the differences in the initial slopes of the PFP yield reduction curves. 
TABLE 2. Comparison of BAP and HAP titers from four replicate single HM passages of TK/OR/71-NS1 $1_{1-230}$ in primary CEK cells starting with a pool of an egg-derived LM-passage stock

\begin{tabular}{|c|c|c|c|c|c|}
\hline \multirow{2}{*}{ Type of passage } & \multirow{2}{*}{ Trial no. } & \multicolumn{3}{|c|}{ Particle titer $/ \mathrm{ml}$ (\% total biologically active particles) ${ }^{a}$} & \multirow{2}{*}{$\mathrm{HAP} / \mathrm{ml}$} \\
\hline & & iCKP (PFP) & niCKP & DIP & \\
\hline LM & TK/OR/71-NS1(1-230) & $3.2 \times 10^{8}(8.6)$ & $22 \times 10^{8}(59.1)$ & $12 \times 10^{8}(32.3)$ & $307 \times 10^{8}$ \\
\hline $\mathrm{HM}^{b}$ & $\begin{array}{l}\text { Trial } 1 \\
\text { Trial } 2 \\
\text { Trial } 3 \\
\text { Trial } 4 \\
\text { Avg }\end{array}$ & $\begin{aligned} 0.0056 & \times 10^{8}(0.01) \\
0.00071 & \times 10^{8}(0.001) \\
0.0008 & \times 10^{8}(0.0059) \\
0.0089 & \times 10^{8}(0.021) \\
0.004 & \times 10^{8}(0.0095)\end{aligned}$ & $\begin{array}{l}4.9 \times 10^{8}(8.5) \\
3.0 \times 10^{8}(4.2) \\
1.6 \times 10^{8}(11.8) \\
2.0 \times 10^{8}(4.8) \\
2.9 \times 10^{8}(7.3)\end{array}$ & $\begin{aligned} 53 & \times 10^{8}(91.5) \\
69 & \times 10^{8}(95.8) \\
12 & \times 10^{8}(88.2) \\
40 & \times 10^{8}(95.2) \\
43.5 & \times 10^{8}(92.7)\end{aligned}$ & $\begin{array}{l}203 \times 10^{8} \\
203 \times 10^{8} \\
121 \times 10^{8} \\
154 \times 10^{8} \\
170 \times 10^{8}\end{array}$ \\
\hline
\end{tabular}

${ }^{a}$ PFP(iCKP $)+$ niCKP + DIP.

${ }^{b}$ Each monolayer of $3 \times 10^{6} \mathrm{CEK}$ cells received $150 \mu \mathrm{l}$ of the LM passage virus.

tectable amounts of DIP $\left(<10^{7} \mathrm{DIP} / \mathrm{ml}\right.$.). Table 1 also compares the PFP, CKP, DIP, and HAP titers of the nine isolates, and Fig. 3 illustrates the broad range of responses in the PFP yield reduction curves when the amount of virus being tested for DIP was standardized on the basis of the HAP (physical particle) content (lower abscissa). Clearly, the greatest efficiency of DIP-mediated interference is achieved at multiplicities where only one or a few particles coinfect a cell with standard virus. Higher multiplicities of DIP often resulted in a disproportionately lower reduction in PFP yield than expected (Fig. 1 and 3). This indicates that a high $\mathrm{m}_{\text {DIP }}$ may interfere with DIP action, possibly because the full-size genomic segments contributed by coinfecting biologically inactive HAP outcompete the corresponding small DI-RNAs delivered by DIP. Similar results were noted with VSV DIP-mediated interference (36).

Comparison of subpopulations of PFP(iCKP), niCKP, DIP, and HAP generated from replicate single-HM passages of a common LM-passage stock of TK/OR/71-NS1 $1_{1-230}$. To gain insight into the relationship of niCKP to DIP, the stock of LM-passage variant TK/OR/71-NS1 $1_{1-230}$ with high titers of particles (Table 2) was subjected to HM passage in CEK cells. This was achieved by infecting four replicate monolayers, each containing an average of $3.0 \times 10^{6} \mathrm{CEK}$ cells with $150 \mu \mathrm{l}$ of the stock. Thus, each cell in the monolayer received an average of 1,705 total HAP. These consisted of 16 PFP(iCKP), 110 niCKP, 60 DIP, and 1,535 HAP with no known biological activity. This analysis shows that although BAP constituted $10 \%$ of the input virus particles, only $10 \%$ of the BAP (i.e., $1 \%$ of the total particle population) were infectious. If we assume that each HAP (physical particle) contained an average of eight gene segments, at an $\mathrm{m}_{\mathrm{HAP}}$ of 1,535 , each cell in the population was exposed to an estimated input of $\sim 12,280$ gene segments. We postulate that such a large influx of gene segments may affect replication and packaging events during the life cycle of the virus (see the Discussion). Based on these multiplicities, virtually all of the cells were coinfected with PFP and DIP. Considering that each replicate sample in Table 2 contained a total of $2 \mathrm{ml}$, the mean ( \pm the standard deviation) yields per cell of the four classes of particles tested were as follows: PFP(iCKP), $0.27 \pm 0.27$; niCKP, $192 \pm 98$; DIP, $2,900 \pm 1,608$; and HAP, $11,350 \pm 2,677$. The HAP/BAP ratio was 1.3 , indicating that BAP constituted ca. $40 \%$ of the total particle yield, with the remaining $60 \%$ representing HAP with no known biological activity. In comparison, the original LMpassage input population consisted of 30\% BAP and 70\% HAP with no known biological activity. Relative to the original LM passage of virus, on average, the following population changes were recorded for the four replicates of a single HM passage: PFP(iCKP), 905-fold decrease; niCKP, 8-fold decrease; DIP, 3.6-fold increase; and HAP, 1.8-fold decrease.

Population dynamics of BAP and HAP generated during each of three successive HM passages starting with a LM passage stock of TK/OR/71-NS1 1-124. . Further insight was sought into the relationship of the two noninfectious subpopulations, niCKP and DIP, by starting with delNS1(1-124), a virus population that lacked detectable niCKP and DIP, and subjecting it to three successive HM passages. The content of these particles was measured after each passage. Table 3 documents a marked decrease in the PFP(iCKP) subpopulation

TABLE 3. Population analysis of the appearance of BAP and HAP in three serial HM passages of TK/OR/71-delNS1 1 -124 in MDCK cells starting from a single pool of LM-passage egg-derived virus

\begin{tabular}{|c|c|c|c|c|c|c|c|}
\hline \multirow{2}{*}{$\begin{array}{l}\text { No. of HM } \\
\text { passages }^{a}\end{array}$} & \multicolumn{4}{|c|}{ Particle titer/ml (avg particle yield/cell) ${ }^{b}$} & \multicolumn{3}{|c|}{ Ratio } \\
\hline & PFP (iCKP) & niCKP & DIP & HAP & DIP/niCKP & $\mathrm{HAP} / \mathrm{BAP}$ & $\mathrm{HAP} / \mathrm{PFP}$ \\
\hline 0 & $6.9 \times 10^{8}(60)$ & $<0.1 \times 10^{8 c}$ & $<0.1 \times 10^{8}(<0.5)^{c}$ & $205 \times 10^{8}(1,783)$ & 1 & 29.7 & 29.7 \\
\hline 1 & $0.113 \times 10^{8}(9.4)^{d}$ & $6.9 \times 10^{8}(580)$ & $15 \times 10^{8}(1,250)$ & $205 \times 10^{8}(17,083)^{c}$ & 2.2 & 9.3 & $1,814.2$ \\
\hline 2 & $0.077 \times 10^{8}(6.4)$ & $4.1 \times 10^{8}(340)$ & $15 \times 10^{8}(1,250)$ & $51 \times 10^{8}(4,250)$ & 3.7 & 2.7 & 662.3 \\
\hline 3 & $0.002 \times 10^{8}(0.17)$ & $1.4 \times 10^{8}(120)$ & $15 \times 10^{8}(1,250)$ & $77 \times 10^{8}(6,417)$ & 10.4 & 4.7 & $38,500.0$ \\
\hline
\end{tabular}

${ }^{a}$ The "0-passage" virus is a pool of allantoic fluids from 9-day-old eggs infected with $\sim 10^{3}$ PFP of an LM passage. Monolayers of $2.4 \times 10^{6}$ MDCK cells received $150 \mu \mathrm{l}$ from the preceding passage.

${ }^{b}$ Values in parentheses were estimated from the total number of cells in the chorioallantoic membrane (6) and the amount of allantoic fluid in 9-day-old eggs.

${ }^{c}$ Less than the level of detection by the assay.

${ }^{d}$ Based on $2.0 \mathrm{ml}$ of virus from $2.4 \times 10^{6}$ MDCK cells in a monolayer; e.g., $\left(0.113 \times 10^{8}\right) \times 2=0.226 \times 10^{6} \mathrm{PFP} \div 2.4 \times 10^{6}=9.4$

${ }^{e}$ Comparable yields have been reported from MDCK cells (35). 


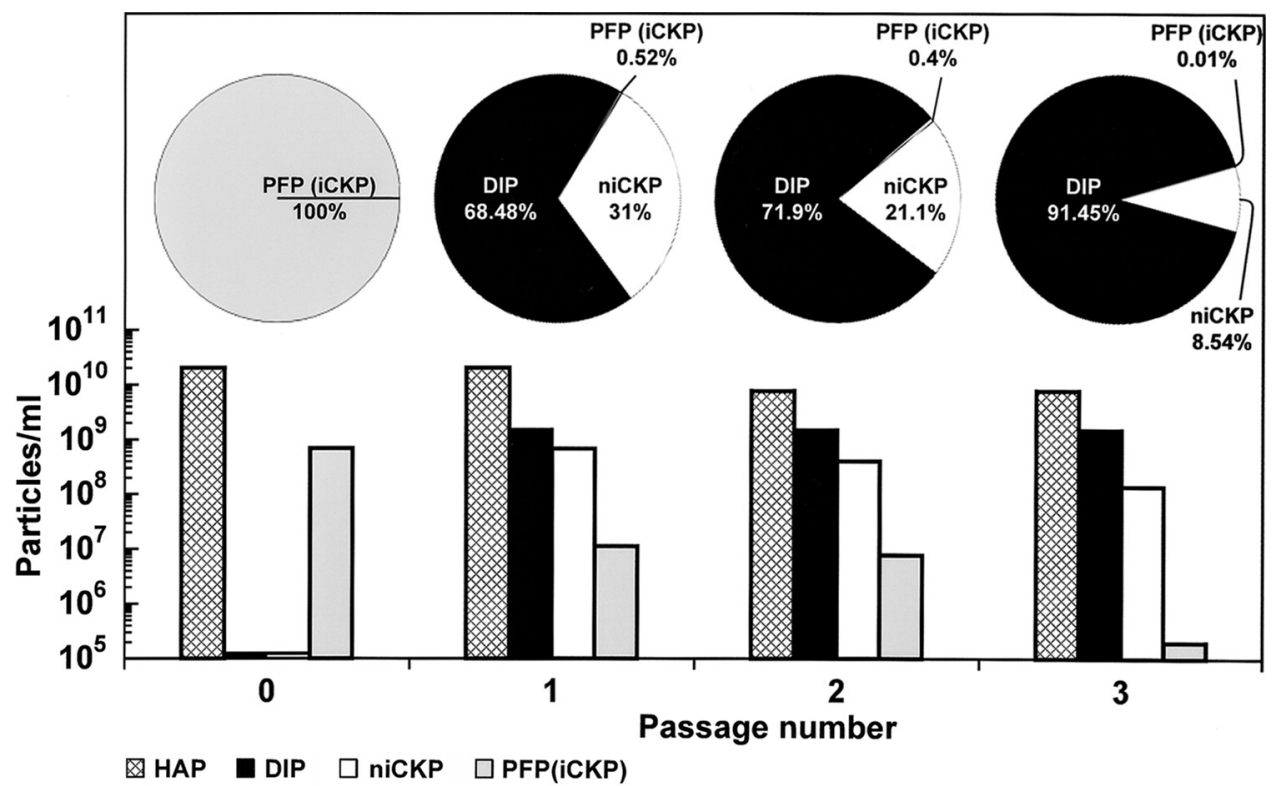

FIG. 4. Population dynamics of BAP and HAP as a function of serial HM passage in MDCK cells. Data taken from Table 3 are presented as histograms that depict changes in the three measurable parameters of BAP-PFP(iCKP), niCKP, and DIP-and of HAP for variant TK/OR/71delNS1 $1_{1-124}$. The 0 passage on the abscissa represents the initial LM passage of egg-derived stock where PFP(iCKP) are the only detectable BAP. The niCKP and DIP appear first at the initial HM passage and are represented in the pie charts as a percentage of the total BAP subpopulations at each successive HM passage.

after the first HM passage and the continued loss of infectivity at each successive passage. This was accompanied by the coincident appearance and maintenance of large numbers of DIP. Most notably, niCKP emerged as a new subpopulation in the first HM passage, making up $31 \%$ of the BAP family, seemingly at the expense of PFP(iCKP) which, in numbers, appeared to be replaced by the niCKP subpopulation (Fig. 4). This suggested that PFP(iCKP) may represent a transitional state to niCKP (see the Discussion). Once generated, the niCKP subpopulation decreased slightly at each successive HM passage from 31 to $21 \%$ to $8.5 \%$. DIP, on the other hand, increased from $69 \%$ to 72 to $91 \%$ during the three passages. These contrasting behaviors suggest that niCKP may represent a transitional state to DIP (see the Discussion and Fig. 4). HAP titers remained relatively high in all passages. Also included in Table 3 (in parentheses) are the average particle yields per cell. The dynamics of these changes are presented in Fig. 4, both as a histogram representing the actual titers of each subpopulation of BAP, and as pie charts representing the percentage of each class in the total BAP population. In all cases the BAP are subsumed within the HAP population. Similar population changes were observed in CEK cells (data not shown).

DIP do not prevent the expression of niCKP. Figure 5A provides qualitative microscopic evidence that a population rich in DIP and which has been UV irradiated $\left(390 \mathrm{ergs} / \mathrm{mm}^{2}\right)$ to inactivate residual PFP and $90 \%$ of its CKP activity while retaining virtually all of its DIP activity does not express any intrinsic capacity to induce apoptosis and kill Vero cells (cf. Fig. 5Aa and b). Nor does it prevent the expression of cell killing by standard virus $(\mathrm{PFP}=\mathrm{iCKP})$ (cf. Fig. 5Ac and d). Similar results were obtained with MDCK cells as host (data not shown). Figure 5B demonstrates quantitatively that DIP that have retained the capacity to interfere with the replication of infectivity (PFP; the lower curve in the figure) have no effect on cell-killing by PFP(iCKP) or niCKP (the upper two curves). Because DIP prevent virus replication, these data demonstrate that infectious particles of influenza virus need not complete a replication cycle to kill a cell, a fact borne out by the cell-killing prowess of the noninfectious niCKP (29) and further implicating their relatedness (see the Discussion).

The UV target sizes for PFP, iCKP, niCKP, and DIP activities. UV target analysis of the biological activities expressed phenotypically by influenza virus is possible because each of the viral gene segments is expressed independently of the others (1). This allows an assessment of the size of the gene segment that is rate limiting for the expression of a particular phenotype. Figure 6 illustrates the survival curves for the four quantifiable phenotypes represented by the biologically active subpopulations of influenza virus particles reported here. Virus stocks containing subpopulations of particles expressing these activities were irradiated with UV (254 nm) light, and the surviving activity was measured as a function of UV dose (ergs $/ \mathrm{mm}^{2}$ ). The UV survival curves for iCKP, niCKP, and PFP were taken from our earlier study (29) and are represented by dashed lines in Fig. 6. The curves for both iCKP and niCKP are coincident (29). The DIP survival curve with data points was determined during the present study and is similar to that reported first by Nayak et al. (26) and confirmed by others (19). These survival curves are assembled here for the first time in a single figure to make clear the distinct genetic requirements between the particles expressing the four different phenotypes. Thus, a single hit to any of the eight unique genes in a PFP (iCKP) inactivates infectivity and apparently converts it into niCKP. Both cell-killing phenotypes can be expressed until 

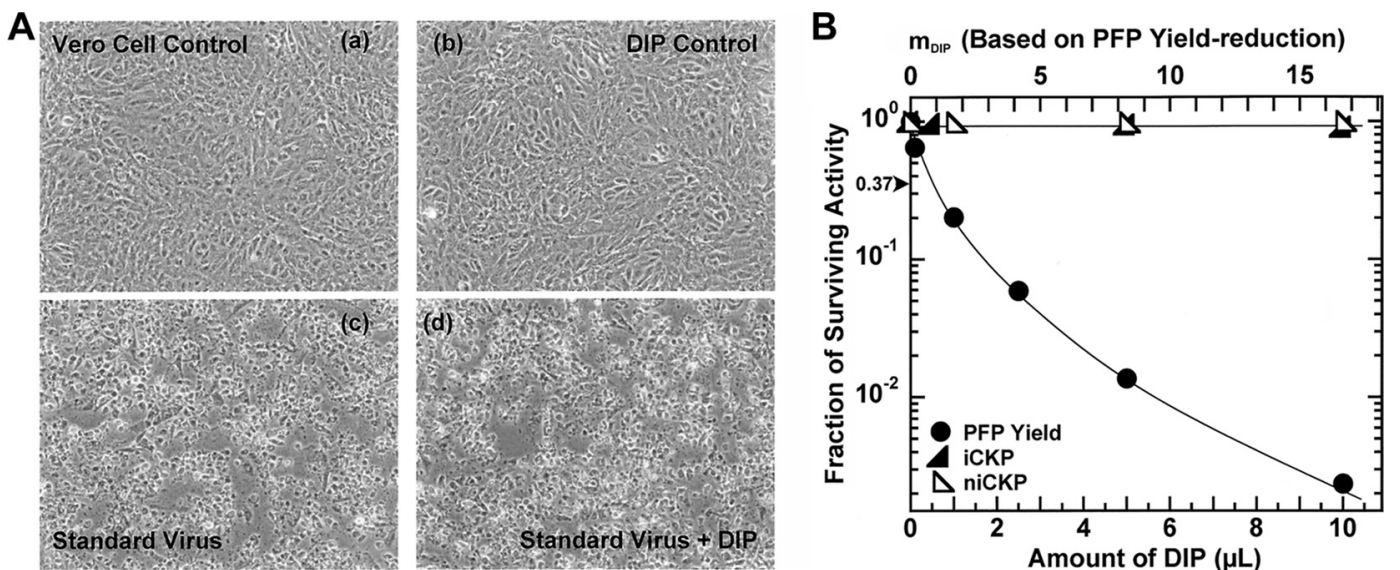

FIG. 5. (A) Effect of DIP on the iCKP (apoptosis-inducing particle) activity of variant TK/OR/71-delNS1 $1_{1-124}$ in monolayers of Vero cells. (a) Uninfected; (b) infected with an $\mathrm{m}_{\text {DIP }}$ of 5; (c) infected with the standard virus only, variant-delNS1 $1_{1-124}$, at an $\mathrm{m}_{\mathrm{PFP}(\mathrm{iCKP})}$ of 3 ; (d) coinfected with standard virus at an $\mathrm{m}_{\mathrm{PFP}(\mathrm{iCKP})}$ of 3 and an $\mathrm{m}_{\mathrm{DIP}}$ of 5 . Virtually complete cytopathic effects (apoptosis) were recorded photographically $24 \mathrm{~h}$ postinfection in both panels c and d. (B) Effect of DIP on PFP yield reduction and CKP (cell killing) by the standard virus. The lower abscissa represents the amount of DIP. The upper abscissa represents the $\mathrm{m}_{\text {DIP }}$ as calculated from the PFP yield reduction curve. The upper two curves show no reduction in iCKP and niCKP activities in cells simultaneously exposed to DIP under conditions where virus replication is compromised (lower curve). The iCKP were derived from variant TK/OR/71-delNS1 $1_{1-124}$ stock which did not have residual niCKP activity. The niCKP were derived from a stock of variant TK/OR/71-NS1 $1_{1-23}$ that originally had an niCKP/iCKP ratio of 7 . This stock was UV irradiated to inactivate $99.97 \%$ of PFP(iCKP) and $75 \%$ of niCKP. Thus, virtually all of the cell killing registered after this treatment was from niCKP.

a gene the size of one particular polymerase subunit is inactivated. The closeness in size of the three-polymerase subunit genes does not allow resolution by UV target analysis of which polymerase gene is rate limiting.

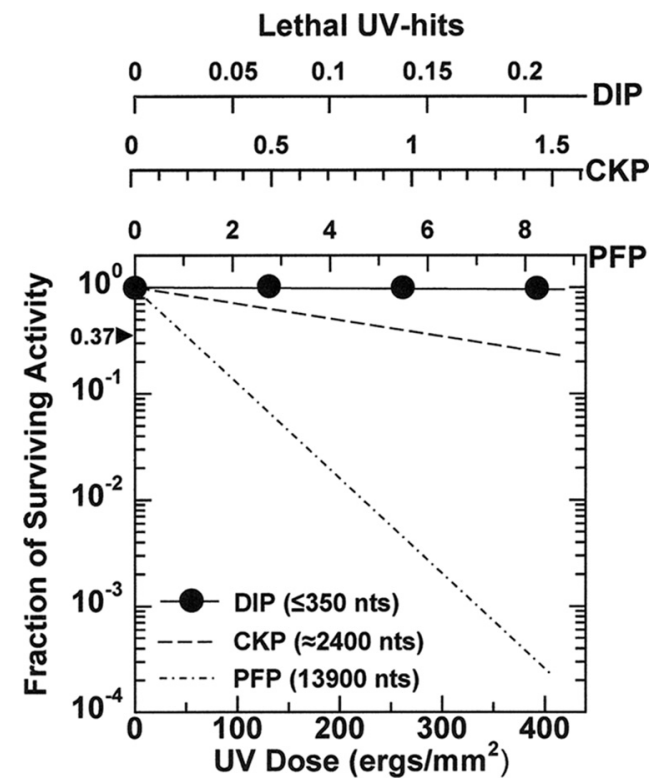

FIG. 6. Comparison of PFP, CKP (iCKP or niCKP), and DIP survival curves as a function of UV dose (lower abscissa). One lethal UV hit to each activity is based on the dose required to achieve 0.37 survival level and was used to generate the three upper abscissas. The resistance of DIP to UV radiation is so high that the 0.37 survival level is not reached within this range of doses. Exposure of DIP to much higher doses revealed a 0.37 survival level at $3,206 \mathrm{ergs} / \mathrm{mm}^{2}$ (data not shown), a finding consistent with a target of $\sim 350 \mathrm{nt}$.

\section{DISCUSSION}

Influenza virus kills cells by apoptosis $(17,40)$, a process presumed to require the replication of infectious virus (PFP). In all cases, the CKP being measured is assumed to be infectious $(\mathrm{PFP}=\mathrm{iCKP})$. However, in addition to PFP(iCKP), the clonogenic assay revealed a new class of influenza virus particles present in excess of infectious particles, those that kill cells in the absence of a productive infection. These were termed niCKP (29). Of the nine isolates tested, six showed titers of niCKP ranging from $3.5 \times 10^{8}$ to $19 \times 10^{8} / \mathrm{ml}$, with a mean niCKP/PFP ratio of 5.9 (Table 1). These six isolates also contained substantial titers of DIP, ranging from $8.6 \times 10^{8}$ to $48 \times 10^{8} / \mathrm{ml}$, with a mean DIP/PFP ratio of 22 . This latter observation was unexpected since all isolates presumably had been maintained by LM passages in eggs and contained high titers of PFP $\left(\right.$ mean titer $\left.=1.2 \times 10^{8} / \mathrm{ml}\right)$. It suggests that high titers of infectious virus may not be sufficient cause to rule out the presence of DIP in stocks of influenza virus. Thus, DIP may also be a prognosticator of the presence of niCKP, as discussed below.

Because niCKP appeared so frequently along with DIP (Table 1), it became important to determine whether these represented subpopulations of distinct particles, or a single particle that could express two different phenotypes. To help resolve this situation the appearance of niCKP was monitored after replicate single passages of one of the isolates, TK/OR/ 71-NS1 $1_{1-230}$, at HM, a sine qua non for the generation of DIP $(26,41)$. Table 2 shows that each replicate HM passage generated both niCKP and DIP, with the average yield of DIP $\sim 13$-fold greater than niCKP on a per-cell basis. The appearance of niCKP and DIP was accompanied by a 905 -fold reduction in PFP content, presumably due to the action of the DIP. This meant that, on average, only about 1 in 150 cells produced 
a PFP. Consequently, DIP and niCKP constituted ca. 93 and $7 \%$ of the total BAP population, respectively.

Clearly, niCKP coappear with DIP, but as a relatively minor fraction of the BAP population. To gain further insight into this relationship, an LM-passage population of variant delNS1 $1_{(1-124)}$ with no detectable levels of niCKP or DIP was subjected to three serial passages at HM (Table 3 and Fig. 4). Both niCKP and DIP were generated in large amounts after a single HM passage. The niCKP appeared, seemingly at the loss of the PFP, particle for particle, and decreased in titer $\sim 2$-fold at each successive HM passage. About twice as much DIP as niCKP appeared after the first HM passage and remained the same at each successive passage. This resulted in a DIP/niCKP ratio of 10 by the third passage. Once again, these DIP and niCKP made up ca. 92 and $8 \%$ of the BAP populations.

During the replicate $\mathrm{HM}$ passages of TK/OR/71-NS1 $1_{1-230}$, input multiplicities of the different classes of particles were as follows: $\mathrm{m}_{\mathrm{PFP}(\mathrm{iCKP})}=16 ; \mathrm{m}_{\text {niCKP }}=110 ; \mathrm{m}_{\mathrm{DIP}}=60$; and $m_{\text {HAP }}=1,535$ (Table 2 ). Thus, each cell received an average of 186 BAP and 1,349 HAP with no known biological activity. Only ca. $1 \%$ of the particles were infectious. Although considered biologically "inactive," the noninfectious HAP have the potential to deliver a large number of gene segments to each cell, e.g., $1,535 \times 8=12,280$. Although it is not likely that all of these particles enter the cell because of neuraminidasemediated elution, saturation of sialic acid receptors, inactive fusion peptide, or some other mechanism(s), it seems likely that a large pool of gene segments may be added to a cell during infection at high multiplicities or as virus accumulates during the multiple cycles that accompany natural infection. We postulate that the presence of large copy numbers of gene segments may perturb two otherwise finely tuned events in the replication of the virus: (i) the temporally regulated replicative steps that normally follow infection at relatively low multiplicities $(24,38)$ and (ii) the selective packaging of the eight unique genes required for infectivity $(16,28)$. We further postulate that such HM-driven aberrations may play a role in generating niBAP.

Collectively, the following data demonstrate that the niCKP and DIP phenotypes are expressed by two distinct subpopulations of particles. (i) The titers of niCKP and DIP in a given stock of influenza virus differ significantly, with the latter usually in $\sim 10$-fold excess over the former. (ii) During each successive HM passage the niCKP subpopulation decreased slightly while that of DIP remained relatively constant. (iii) Unlike niCKP, DIP do not kill cells, as observed microscopically (Fig. 5). (iv) DIP do not interfere with the cell-killing capacity of iCKP or niCKP (Fig. 5), indicating that DIP do not affect the synthesis of a yet-to-be-defined cell-killing factor, even though they block the replication of a coinfecting standard particle (PFP) (Fig. 5B). (v) UV target analyses distinguish between the minimum genetic requirements for expression of the niCKP and DIP phenotypes (Fig. 6). Thus, expression of DIP requires the presence of a small RNA, the DI RNA ( $\sim 350$ nucleotides [nt]), a subgenomic RNA derived from a polymerase gene (26). In contrast, niCKP requires the expression of a larger RNA, $\sim 2,400 \mathrm{nt}$, the equivalent of a specific polymerase subunit gene segment (29). If inactivation of any one of the three polymerase subunit genes sufficed to inactivate niCKP then the target of inactivation would be the sum of the three genes $(\sim 6,900 \mathrm{nt})$, resulting in a survival curve with twice the slope of that for PFP ( 13,600 nt) (33). This was not observed. We infer that preferential inactivation of a specific polymerase subunit gene represents the rate-limiting step in the expression of niCKP. The fact that iCKP, but not PFP, share the same UV target as niCKP is interpreted to mean that a UV hit to any of the eight unique genes suffices to inactivate infectivity (1) while maintaining the cell-killing capacity of the particle until a particular polymerase subunit gene is inactivated (29), possibly the PA gene $(15,32)$. Thus, it appears that iCKP can be converted to niCKP through the UV inactivation of at least one of the five nonpolymerase genes if the specific polymerase subunit gene that is required to express the CKP phenotype remains intact.

Stocks of both strains of $\mathrm{PR} / 8 / 34$, along with TK/OR/71 variant delNS1 $1_{1-124}$ (Table 1 ) had low or undetectable amounts of niCKP and/or DIP compared to the other strains studied. That both of the PR/8/34 isolates expressed full-size NS1 suggests that the propensity to generate these two niBAP does not simply relate to the truncation of the NS1 protein. Suffice it to note at this stage that all three isolates generate DIP after infection at high multiplicities (data not shown). The genetic components that govern generation of niCKP and DIP are currently being assessed.

In an earlier study, Carter and Mahy (7) inferred the presence of defective noninterfering particles (DNIP) in stocks of influenza virus enriched for DIP by HM passages. The inference was based on an excess activity of virion-associated RNA polymerase, measured in vitro, relative to that expected from the presence of the low numbers of infectious virus in these stocks. Even stocks from LM passages displayed an excess in polymerase activity over that expected from the content of infectious virus (7). Since niCKP display these same attributes, i.e., require functional polymerase, are noninfectious, lack defective-interfering particle activity, and also are present in both HM and LM passages of virus, we propose that DNIP are the equivalent of niCKP.

Based on the genetic requirements defined by UV target analysis, the dynamics of the changes in subpopulations observed as they proceed through successive HM passages, and recognizing that all BAP are subsumed in the HAP population, we postulate that at least four types of virus particles are produced as a consequence of infecting cells with influenza virus, and the magnitude of each subpopulation is highly dependent upon the multiplicity of infection that generates them. The four types, presented in the order of decreasing genomic complexity required to express their phenotype, were as follows: $\mathrm{PFP}(\mathrm{iCKP}) \rightarrow$ niCKP(DNIP $) \rightarrow$ DIP $\rightarrow$ HAP.

There are other subpopulations of BAP in influenza virus stocks not yet accounted for in populations with defined numbers of PFP, niCKP, DIP, and HAP. Thus, IFP $(20,21)$ and ISP $(21,23)$ display mutually exclusive phenotypes that may be present in 20-fold excess over PFP (21). Ongoing studies are designed to determine the extent to which these phenotypes represent independent or overlapping subpopulations.

Lastly, the observations that DIP found in natural influenza of chickens (9) can suppress the pathogenic effects of virulent virus (4), the promising role of DIP as an effective prophylactic and therapeutic antiviral agent $(11,12)$ and that isolates of LPAI which contain truncated NS1 genes ameliorate patho- 
genesis (8), induce large amounts of IFN and serve as liveattenuated vaccines $(14,34,39,42)$, and may affect apoptosis and the dynamics of virus production for vaccines (35) encourage us to study further the relationship of PFP, niCKP, DIP, HAP, IFP, and ISP in order to better understand the role these subpopulations play in the pathogenesis and evolution of influenza virus, the formulation of live-attenuated vaccines, and vaccine production.

\section{ACKNOWLEDGMENTS}

This research was supported in part by USDA grants 58-1940-0-007 through the Center for Excellence in Vaccine research at the University of Connecticut and a USDA SCA award 58-6612-7-157. This study benefited from the services of the Animal Cell Culture Facility of the Biotechnology-Bioservices Center of the University of Connecticut.

\section{REFERENCES}

1. Abraham, G. 1979. The effect of ultraviolet radiation on the primary transcription of influenza virus messenger RNAs. Virology 97:177-182.

2. Akkina, R. K., T. M. Chambers, and D. P. Nayak. 1984. Mechanism of interference by defective-interfering particles of influenza virus: differential reduction of intracellular synthesis of specific polymerase proteins. Virus Res. 1:687-702.

3. Basler, C. F., and P. V. Aguilar. 2008. Progress in identifying virulence determinants of the 1918 H1N1 and the Southeast Asian H5N1 influenza A viruses. Antivir. Res. 79:166-178.

4. Bean, W. J., Y. Kawaoka, J. M. Wood, J. E. Pearson, and R. G. Webster. 1985. Characterization of virulent and avirulent A/Chicken/Pennsylvania/83 influenza viruses: potential role of defective interfering RNAs in nature. J. Virol. 54:151-160.

5. Bellett, A. J. D., and P. D. Cooper. 1959. Some properties of the transmissible interfering component of vesicular stomatitis preparations. J. Gen. Microbiol. 21:498-509.

6. Cairns, H. J. F., and S. Fazekas de St. Groth. 1957. The number of allantoic cells in the chick embryo. J. Immunol. 78:191-200.

7. Carter, M. J., and B. W. J. Mahy. 1982. Incomplete avian influenza virus contains a defective noninterfering component. Arch. Virol. 71:12-25.

8. Cauthen, A. N., D. E. Swayne, M. J. Sekellick, P. I. Marcus, and D. L Suarez. 2007. Amelioration of influenza virus pathogenesis in chickens attributed to the enhanced interferon-inducing capacity of a virus with a truncated NS1 gene. J. Virol. 81:1838-1847.

9. Chambers, T. M., and R. G. Webster. 1987. Defective interfering virus associated with $\mathrm{A} /$ Chicken/Pennsylvania/83 influenza virus. J. Virol 61:1517-1523

10. Desselberger, U. 1975. Relation of virus particle counts to the hemagglutinating activity of influenza virus suspensions measured by the HA pattern test and by the photometric HCU method. Arch. Virol. 49:365-372.

11. Dimmock, N. J., S. Beck, and L. McLain. 1986. Protection of mice from lethal influenza: evidence that defective interfering virus modulates the immune response and not virus multiplication. J. Gen. Virol. 67:839-850.

12. Dimmock, N. J., E. W. Rainsfors, P. D. Scott, and A. C. Marriott. 2008. Influenza virus protecting RNA: an effective prophylactic and therapeutic antiviral. J. Virol. 82:8570-8578.

13. Donald, H. B., and A. Isaacs. 1954. Electron microscope counts of influenza virus particles. J. Gen. Microbiol. 10:457-464.

14. Donelan, N. R., C. F. Basler, and A. García-Sastre. 2003. A recombinant influenza virus expressing an RNA-binding-defective NS1 protein induces high levels of beta interferon and is attenuated in mice. J. Virol. 77:1357-1366.

15. Fodor, E., L. J. Mingay, M. Crow, T. Deng, and G. G. Brownlee. 2003. A single amino acid mutation in the PA subunit of the influenza virus RNA polymerase promotes the generation of defective interfering RNAs. J. Virol 77:5017-5020.

16. Fujii, Y., H. Goto, T. Watanabe, T. Yosida, and Y. Kawaoka. 2003. Selective incorporation of influenza virus RNA segments into virions. Proc. Natl. Acad. Sci. USA 100:2002-2007.

17. Hinshaw, V. S., C. W. Olsen, N. Dybdahl-Sissoko, and D. Evans. 1994 Apoptosis: a mechanism of cell killing by influenza A and B viruses. J. Virol. 68:3667-3673.

18. Janda, J. M., A. R. Davis, D. P. Nayak, and B. K. De. 1979. Diversity and generation of defective interfering influenza virus particles. Virology 95:48-58.

19. Kantorovich-Prokudina, E. N., N. P. Semyonova, O. N. Berezina, and V. M Zhdanov. 1980. Gradual changes of influenza virions during passage of undiluted material. J. Gen. Virol. 50:23-31.
20. Marcus, P. I. 1982. Interferon induction by viruses. IX. Antagonistic activities of virus particles modulate interferon production. J. Interferon Res. 2:511-518

21. Marcus, P. I., J. M. Rojek, and M. J. Sekellick. 2005. Interferon induction and/or production and its suppression by influenza viruses. J. Virol. 79:2880-2890.

22. Marcus, P. I., and M. J. Sekellick. 1974. Cell killing by viruses. I. Comparison of cell-killing, plaque-forming, and defective-interfering particles of vesicular stomatitis virus. Virology 57:321-328.

23. Marcus, P. I., and M. J. Sekellick. 1985. Interferon induction by viruses. XIII. Detection and assay of interferon induction-suppressing particles. Virology 142:411-415

24. Min, J.-Y., S. Li, G. C. Sen, and R. M. Krug. 2007. A site on the influenza virus NS1 protein mediates both inhibition of PKR activation and tempora regulation of viral RNA. Virology 363:236-243.

25. Nakajima, K., and A. Sugiura. 1977. Three-factor crosses of influenza virus. Virology 81:486-489.

26. Nayak, D. P., T. M. Chambers, and R. K. Akkina. 1985. Defective-interfering (DI) RNAs of influenza viruses: origin, structure, expression, and interference. Curr. Top. Microbiol. Immunol. 114:103-151.

27. Nayak, D. P., K. Tobita, J. M. Janda, A. R. Davis, and B. K. De. 1978 Homologous interference mediated by defective-interfering influenza virus derived from a temperature-sensitive mutant of influenza virus. J. Virol. 28:375-386.

28. Neumann, G., T. Wantanabe, H. Ito, S. Watanabe, H. Goto, Peng Gao, M. Hughes, D. R. Perez, R. Donis, E. Hoffman, G. Hobom, and Y. Kawaoka. 1999. Generation of influenza A viruses entirely from cloned cDNAs. Proc. Natl. Acad. Sci. USA 96:9345-9350.

29. Ngunjiri, J. M., M. J. Sekellick, and P. I. Marcus. 2008. Clonogenic assay of type A influenza viruses reveals noninfectious cell-killing (apoptosis-inducing) particles. J. Virol. 82:2673-2680.

30. Noble. S., L. McLain, and N. J. Dimmock. 2004. Interfering vaccine: a novel antiviral that converts a potentially virulent infection into one that is subclinical and immunizing. Vaccine 22:3018-3025.

31. Norton, G. P., T. Tanaka, K. Tobita, S. Nakada, D. A. Buonagurio, D. A Greenspan, M. Krysta, and P. Palese. 1987. Infectious influenza virus A and $B$ virus variants with long carboxyl-terminal deletions in the NS1 polypeptides. Virology 156:204-213.

32. Odagiri, T., and K. Tobita. 1990. Mutation in NS2, a nonstructural protein of influenza virus, extragenically causes aberrant replication and expression of the PA gene and leads to generation of defective interfering particles. Proc. Natl. Acad. Sci. USA 87:5988-5992.

33. Palese, P., and M. L. Shaw. 2007. Orthomyxoviridae: the viruses and their replication, p. 1646-1689. In D. M. Knipe, P. M. Howley, D. E. Griffin, R. A. Lamb, M. A. Martin, B. Roizman, and S. E. Straus (ed.), Fields virology, 5th ed. Lippincott-Raven Publishers, Philadelphia, PA.

34. Quinlivan, M., D. Zmerin, A. García-Sastre, A. Cullinane, T. Chambers, and P. Palese. 2005. Attenuation of equine influenza viruses through truncations of the NS1 protein. J. Virol. 79:8431-8439.

35. Schulze-Horsel, J., M. Schulze, G. Agalaridis, Y. Genzel, and U. Reichl. 2009. Infection dynamics and virus-induced apoptosis in cell culture-based influenza vaccine production: flow cytometry and mathematical modeling. Vaccine 27:2712-2722.

36. Sekellick, M. J., and P. I. Marcus. 1980. Viral interference by defective particles of vesicular stomatitis virus measured in individual cells. Virology 104:247-252.

37. Sekellick, M. J., and P. I. Marcus. 1986. Induction of high titer chicken interferon. Methods Enzymol. 118:115-125.

38. Shapiro, G. I., T. Gurney, and R. M. Krug. 1987. Influenza virus gene expression: control mechanisms at early and late times of infection and nuclear-cytoplasmic transport of virus-specific RNAs. J. Virol. 61:764-773.

39. Solórzano, A., R. J. Webby, K. M. Lager, B. H. Janke, A. García-Sastre, and J. A. Richt. 2005. Mutations in the NS1 protein of swine influenza virus impair anti-interferon activity and confer attenuation in pigs. J. Virol. 79: 7535-7543.

40. Takizawa, T., S. Matsukkawa, Y. Higuchi, S. Nakamura, Y. Nakanishi, and R. Fukuda. 1993. Induction of programmed cell death (apoptosis) by influenza virus infection in tissue culture cells. J. Gen. Virol. 74:2347-2355.

41. Von Magnus, P. 1954. Incomplete forms of influenza virus. Adv. Virus Res. 2:59-78

42. Wang, L., D. L. Suarez, M. Pantin-Jackwood, M. Mibayashi, A. GarcíaSastre, Y. M. Saif, and C.-W. Lee. 2008. Characterization of influenza virus variants with different sizes of the nonstructural (NS) genes and their potential as a live influenza vaccine in poultry. Vaccine 26:3580-3586.

43. Wright, P. F., G. Neumann, and Y. Kawaoka. 2007. Orthomyxoviruses, p. 1691-1740. In D. M. Knipe, P. M. Howley, D. E. Griffin, R. A. Lamb, M. A. Martin, B. Roizman, and S. E. Straus (ed.), Fields virology, 5th ed. Lippincott-Raven Publishers, Philadelphia, PA.

44. Zhirnov, O. P., A. V. Ovcharenko, and A. C. Bukrinskya. 1983. A modified plaque assay method for accurate analysis of infectivity of influenza viruses with uncleaved hemagglutinin. Arch. Virol. 71:177-183. 